\title{
Experimentally driven atomistic model of 1,2 Polybutadiene
}

Article

Accepted Version

Creative Commons: Attribution 3.0 (CC-BY)

Gkourmpis, T. and Mitchell, G. R. (2014) Experimentally driven atomistic model of 1,2 Polybutadiene. Journal of Applied Physics, 115 (5). 053505. ISSN 0021-8979 doi: https://doi.org/10.1063/1.4863950 Available at https://centaur.reading.ac.uk/35727/

It is advisable to refer to the publisher's version if you intend to cite from the work. See Guidance on citing.

Published version at: http://scitation.aip.org/content/aip/journal/jap

To link to this article DOI: http://dx.doi.org/10.1063/1.4863950

Publisher: American Institue of Physics

Publisher statement: The following article has been accepted by Journal of Applied Physics. After it is published, it will be found at http://scitation.aip.org/content/aip/journal/jap Copyright (year) Author(s). This article is distributed under a Creative Commons Attribution 3.0 Unported License.

All outputs in CentAUR are protected by Intellectual Property Rights law, including copyright law. Copyright and IPR is retained by the creators or other copyright holders. Terms and conditions for use of this material are defined in the End User Agreement.

www.reading.ac.uk/centaur

\section{CentAUR}


Central Archive at the University of Reading

Reading's research outputs online 
The following article has been accepted by the Journal of Applied Physics. After it is published, it will be found at http://scitation.aip.org/content/aip/journal/jap.

Copyright 2014 Thomas Gkourmpis and Geoffrey R. Mitchell

This article is distributed under a Creative Commons Attribution 3.0 Unported License.

\title{
Experimentally driven atomistic model of 1,2 polybutadiene
}

Thomas Gkourmpis ${ }^{\mathrm{a}{ }^{8}}$ and Geoffrey R. Mitchell ${ }^{\mathrm{a}, \mathrm{b}}$

${ }^{a}$ Polymer Science Centre J. J. Thomson Physical Laboratory Department of Physics University of Reading Reading RG6 6AF UK

${ }^{\mathrm{b}}$ Centre for Rapid and Sustainable Product Development Institute Polytechnic Leiria Marinha Grande Portugal

*Corresponding author

$\S$ Present Address: Borealis AB Innovation \& Technology Stenungsund SE 444-86 Sweden thomas.gkourmpis@borealisgroup.com

\begin{abstract}
We present an efficient method of combining wide angle neutron scattering data with detailed atomistic models, allowing us to perform a quantitative and qualitative mapping of the organisation of the chain conformation in both glass and liquid phases. The structural refinement method presented in this work is based on the exploitation of the intrachain features of the diffraction pattern and its intimate linkage with atomistic models by the use of internal coordinates for bond lengths, valence angles and torsion rotations. Atomic connectivity is defined through these coordinates that are in turn assigned by pre-defined probability distributions, thus allowing for the models in question to be built stochastically. Incremental variation of these coordinates allows for the construction of models that minimise the differences between the observed and calculated structure factors. We present a series of neutron scattering data of 1,2 polybutadiene at the region $120-400 \mathrm{~K}$. Analysis of the experimental data yield bond lengths for $\mathrm{C}-\mathrm{C}$ and $\mathrm{C}=\mathrm{C}$ of $1.54 \AA$ and $1.35 \AA$ respectively. Valence angles of the backbone were found to be at $112^{\circ}$ and the torsion distributions are characterised by five rotational states, a three-fold trans-skew \pm for the backbone and gauche \pm for the vinyl group. Rotational states of the vinyl group were found to be equally populated, indicating a largely atactic chan. The two backbone torsion angles exhibit different behaviour with respect to temperature of their trans population, with one of them adopting an almost all trans sequence. Consequently the resulting configuration leads to a rather persistent chain, something indicated by the value of the characteristic ratio extrapolated from the model. We compare our results with theoretical predictions, computer simulations, RIS models and previously reported experimental results.
\end{abstract}

\section{Introduction}

Amorphous polymers are generally considered to be characterised by the absence of long-range order, for example the orientation or translation order found in liquid crystals or the crystalline state respectively is not present. In this state of matter the level of order is concentrated on the vicinity of the polymeric chain and is mostly associated with the atomic connectivity. Thus the chemical structure will dominate the short-range ordering of the chain through the organisation of segments whose continuous assembly represents the polymeric trajectory.

The basic building block of a polymer chain is the chemical bond, and the level of freedom of rotation it enjoys, leads to large number of macromolecular structural arrangements. These continuous assemblies of chemical bonds are free to explore all possible spatial dimensions and the resulting chain trajectory is traditionally treated in terms of rotation states [1,2]. In principle a large number of these states can be considered and the chain segment can be seen as the part of the macromolecule where the chain conformation is assumed to be broadly constant [3]. The size of a segment can vary from a few skeletal bonds $(\sim 5 \AA)$ for polyethylene to much larger distances of $20-30 \AA$ for polycarbonate.

Wide angle X-ray and neutron scattering and nuclear magnetic resonance (NMR) procedures are the most commonly used experimental methods in the study of the short-range order of polymeric liquids and glasses due to their sensitivity of variations to the local structure. NMR spectroscopy is a powerful method in the examination of structure and dynamics of amorphous polymers. Its main advantage lies with the exceptionally available chemical selectivity. Using specific sequences of pulses the broad features of traditional solid state NMR can be decomposed providing a vast and detailed separation of molecular features. The power of NMR is its capability of providing detailed information that is in a very localised level. 
On the contrary scattering methods provide information over a much larger range of length scales, but special models and experimental techniques such as isotropic substitution in neutron scattering are needed if detailed information is required. The measurement of scattering intensities over a broad $Q$ range $(Q=4 \pi \sin \theta / \lambda$, where $2 \theta$ is the scattering angle and $\lambda$ is the incident wavelength) provides information about bond lengths and segmental interactions at distances of approximately $0.1-100 \AA$. Neutrons offer significant advantages over x-rays in the identification of the local structure due to the much higher $Q$ range available and the equivalent increase in real space resolution coupled with the possibility of the variation of scattering lengths via isotropic substitution.

Over the years a wide range of experimental studies on glassy and liquid polymers have been undertaken, with the majority of diffraction studies concentrated in the role of dynamics of the glass transition[4] and the large scale properties probed by small angle neutron scattering (SANS)[5]. Most of the scattering studies of amorphous polymers over an extended $Q$ range probing the details of the local conformation have been made[6,7] using wide angle x-ray scattering techniques. The use of neutrons and the advantage of the extended reciprocal space available have not been used in these polymer systems to the same extent.

The experimentally observed structure factor from both neutrons and x-rays arises from a wide range of correlations. These structural correlations can be separated in to those arising from correlations within the chain (intrachain) and those arising by different chains (interchain)[6]. The interchain correlations provide information on the different ways polymeric segments arrange themselves in the bulk. The intrachain contribution to the diffraction pattern can be extracted from the experimental data at values of the momentum transfer $Q \geq 2-2.5 \AA^{-1}$, using atomistic modelling techniques.

In the past we have developed a Reverse Monte Carlo method for extracting the detailed structural arrangements of amorphous polymers from neutron diffraction patterns[13]. Our method is based on the original Reverse Monte Carlo (RMC) that was developed for modelling liquids and glasses using atomistic models together with diffraction data[14]. The main advantage of such approach is the usage of the actual experimental data (in the form of the diffraction pattern) as constrain in the calculation instead of force field definitions as in traditional molecular dynamics. In our approach we utilised the natural separation observed in a diffraction pattern between inter and intrachain scattering[6,7], thus allowing for the atomic positions and their inherent connectivity to act as constraints in the calculation. In other words the constrain in the Monte Carlo calculation is the atomic connectivity, thus leading to a system where no ambiguities exist and the only driving force is the actual experimental data. This approach has been tested for a number of polymers of different linear architectures and degrees of flexibility with successful results. In this study we are presenting for the first time an evolution of this method in a more complicated system where the chain periodicity is interrupted by double bonds and the existence of side groups.

Polybutadiene is a material vital to the rubber industry with a wide range of technological applications[15]. Using a range of polymerisation techniques, different microstructures of linear $(1,4)$ and vinyl $(1,2)$ chains can be produced in a consistent and reliable manner both in laboratory and industrial conditions. Due to its importance and simple chemical structure, polybutadiene has been studied extensively in the past[16-20] but most of the studies concentrated on the linear 1,4 isomer. Unfortunately the less common 1,2 polybutadiene has not received this kind of attention and there is a lack of published results. Recently Ma and Zhang[21] used rotational isomeric state (RIS) theory to predict the unperturbed radius of gyration of 1,2 polybutadiene. Molecular Dynamics and Molecular Mechanics simulations of isotactic 1,2 polybutadiene have been performed by Mattice[22], and a more recent atomistic Monte Carlo simulation of atactic 1,2 polybutadiene has been presented[23] using a united atom force-field based on quantum mechanical calculations of model compounds[24].

A wide angle neutron scattering study in a temperature range covering almost the entire experimentally accessible liquid and glassy regime for a polybutadiene copolymer reach in 1,2 fraction is presented. Intrachain correlations reflecting the chain conformation were extracted using a structural refinement technique, giving a picture of the changes in the local conformation as a function of temperature. Our findings are compared with previously reported results and computer simulation predictions.

\section{Computational Method}

The computational method used in this study has been presented elsewhere[13] so in this section a very quick overview will be presented for the facilitation of the reader. In a neutron scattering experiment, under the so-called static approximation[30] the measured intensity is proportional to the differential cross section that can be related to the static structure factor $S(Q)$. In the general case of a disordered system comprised of identical $N$ nuclei the differential cross section can be expressed as 


$$
\frac{d \sigma}{d \Omega}=N b^{2} S(Q)
$$

where $b$ is the scattering length of the nuclei and $S(Q)$ is defined as the spatial Fourier transform of the atomic pairwise correlation functions

$$
S(Q)=1+\rho_{o} \int_{0}^{\infty}(g(r)-1) \exp (i Q r) d r
$$

where $\rho_{o}$ is the average density of the system and $g(r)$ is the pair distribution function expressing the probability of an atom to exist at distance $r$ from the origin. Both the radial distribution function and the structure factor are split into partial terms if more than one chemical species are present in the system.

In a broad $Q$ scattering experiment there is an intrinsic difficulty of extracting structural information from the diffuse diffraction pattern. The Fourier Transform of the structure factor can provide information of high confidence, concerning the near neighbour distances, but the intrachain region of the scattering that contains all the information regarding the conformational characteristics remains hidden.

Alternatively extraction of the necessary information can be performed via direct comparison of the experimental data with computer generated statistical models. An artificially obtained structure factor can easily be obtained if all the atomic coordinates of all atoms in the system are known. Therefore by reconstructing and directly comparing the calculated scattering to the actual experimental data a large volume of structural parameters can be probed and their effect on the scattering pattern can be established.

The main problem in simulating a polymer chain arises from the internal rotations, especially around the skeletal bonds. These rotations need to be quantified if one wants to establish a picture of the conformational characteristics of the polymeric chain of interest. These conformational arrangements exist as localised conformations, separated by energy barriers, thus forcing the conformers to undergo a conformational transition when moving from one spatial arrangement to another[25,27].

This picture can be further simplified by the use of the rotational isomeric state (RIS) approach, where for each backbone connection (bond) the continuous distribution of possible spatial rotations (torsion angles) is substituted by a finite number of states (values or positions) that correspond to the conformational energy minima[1, 2]. The resulting chain is represented as an equilibrium mixture of these states, each of which is characterised by the actual state (torsion angle value) and the equivalent probability of occurrence. Generally these probabilities are observed to be conditional on the neighbouring states, but the principle is valid even when no conditionality is applied.

In our method we have generated a fully atomistic model as a single chain of 15,000 skeletal bonds using an in-house developed software package[26]. The atomic connectivity is defined in a set of internal coordinates, leading to a very simple and easy to handle definition for the bond length, valence angle and torsion rotation. The chain is built using a stochastic Monte Carlo method, leading to a number of different initial configurations. The diffraction is computed via the calculation of all the interactomic distances using Debye's relationship[28, 29]

$$
S_{C}(Q)=\frac{1}{N} \sum_{i} \sum_{i \neq j} b_{i} b_{j} \frac{\sin \left(Q r_{i j}\right)}{Q r_{i j}}
$$

where $N$ is the number of atoms, $b_{i}, b_{j}$ are the neutron scattering lengths[30,31] of atoms $i$ and $j$ and $r_{i j}$ is the equivalent interatomic distance. Since we are interested in correlations arising from atoms that are covalently joined, we used a window function to exclude distant correlations that do not contribute significantly to the structure factor and have the adverse effect of extending the calculation time.

The experimental data used in this study are assumed to exhibit just systematic errors arising from the instrumentation. The SANDALS diffractometer experimental resolution $\Delta Q / Q \approx 4 \%$ has been found to be generally constant in all scattering angles[32] something typical of this type of time-of-flight instrument[31]. Consequently the structure factor calculated from the model was smeared with a Gaussian function of width proportional to $Q$ to simulate the experimental resolution. 
The calculated scattering $S_{C}(Q)$ from the model is only of intrachain origin and can be directly compared with the experimental structure factor $S_{E}(Q)$ (only in the part of the curve that corresponds to intrachain correlations) via a statistical $\chi^{2}$ test

$$
\chi^{2}=\frac{1}{n} \sum_{i=1}^{n}\left[Q S_{C}(Q)-Q S_{E}(Q)\right]^{2}
$$

with $n$ the number of points considered. The $\chi^{2}$ test provides information on the impact the different length scales have on the scattering pattern, allowing us to observe the regions most influenced by the different levels of atomic connectivity. Each chosen parameter is changed while all others are kept fixed, allowing for the evaluation of the scattering from a series of different models (configurations). The value of the given parameter that corresponds to the minimum value of the $\chi^{2}$ test is inserted in the model as an initial value and another parameter is searched in an iterative manner. The iteration continues until all parameters are searched and the statistical test result remains practically constant, in a manner essentially equivalent to the Ravine Method[33]. The parameters of the model are those used to define the probability distributions from which values of each component of the internal geometry (see illustration) are drawn stochastically[34, 35]. Consequently for each bond type there is a probability distribution defined by the mean value and the width. These distributions are taken as Gaussian in this work.

From this procedure a three dimensional picture of the local conformation of a polymer chain can be acquired, especially the level of stiffness can be determined by analysing the torsional sequences on the backbone. Although a little simplified, this type of representation is useful as it provides a simple picture of the polymer chain, while at the same time it allows for insights into the possible packing possibilities in the bulk.

\section{Experimental}

Fully deuterated (>99\%) 1,2 polybutadiene $\mathrm{M}_{\mathrm{w}}=77000, \mathrm{M}_{\mathrm{W}} / \mathrm{M}_{\mathrm{n}}=1.08$ was supplied by Polymersource Inc in Canada. Sample characterisation (provided by the supplier) indicated a largely atactic system containing $78 \% 1,2$ and $22 \% 1,4$ polybutadiene sequences. The glass transition was found to be $-13.5^{\circ} \mathrm{C}(259.5 \mathrm{~K})$ by DSC. The sample was dissolved in Cyclohexane and casted into an aluminium container of $1 \mathrm{~mm}$ thickness and $36 \mathrm{~mm}$ diameter. All samples were left to dry under atmospheric pressure and vacuum for 72 hours and solvent evaporation was checked by continuous weighting.

Neutron scattering measurements were performed in the ISIS Pulsed Neutron Source in the UK using SANDALS diffractometer[36]. Temperature control was achieved by a CCR cryogenic unit with temperature fluctuations of the order of $\pm 2^{\circ}$ and all data were collected for a minimum of 500 to a maximum of $3500 \mu \mathrm{A}$ integrated proton current (3.5-15h). Data collected in all 18 detector banks were normalised to the incident neutron beam and calibrated by a standard vanadium sample provided by the facility. Corrections for absorption, multiple and self scattering and attenuation were performed using the data analysis routine provided by the facility[37]. Finally the fully corrected data of each individual detector bank were merged to a total structure factor $S(Q)$.

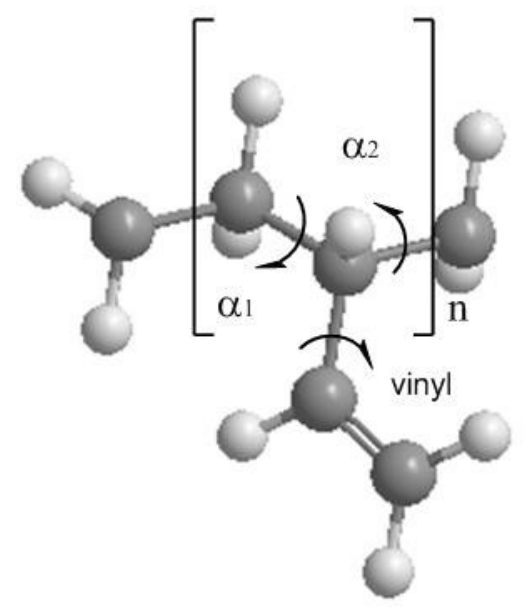

Illustration: Fragment of 1,2 polybutadiene indicating the geometrical parameters of the model

\section{Results and Discussion}




\section{Neutron Scattering}

Neutron scattering data for 1,2 polybutadiene were collected at 11 temperatures ranging $120-400 \mathrm{~K}$ covering almost the entirely experimentally accessible liquid and glassy regime. In Figures 1 and 2 the total structure factors obtained in the study can be seen. These patterns indicate that except for the first scattering peak (Figure 2) the pattern remains largely unchanged for the entire temperature range. The position and intensity of the first scattering peak is seen to change significantly with temperature.

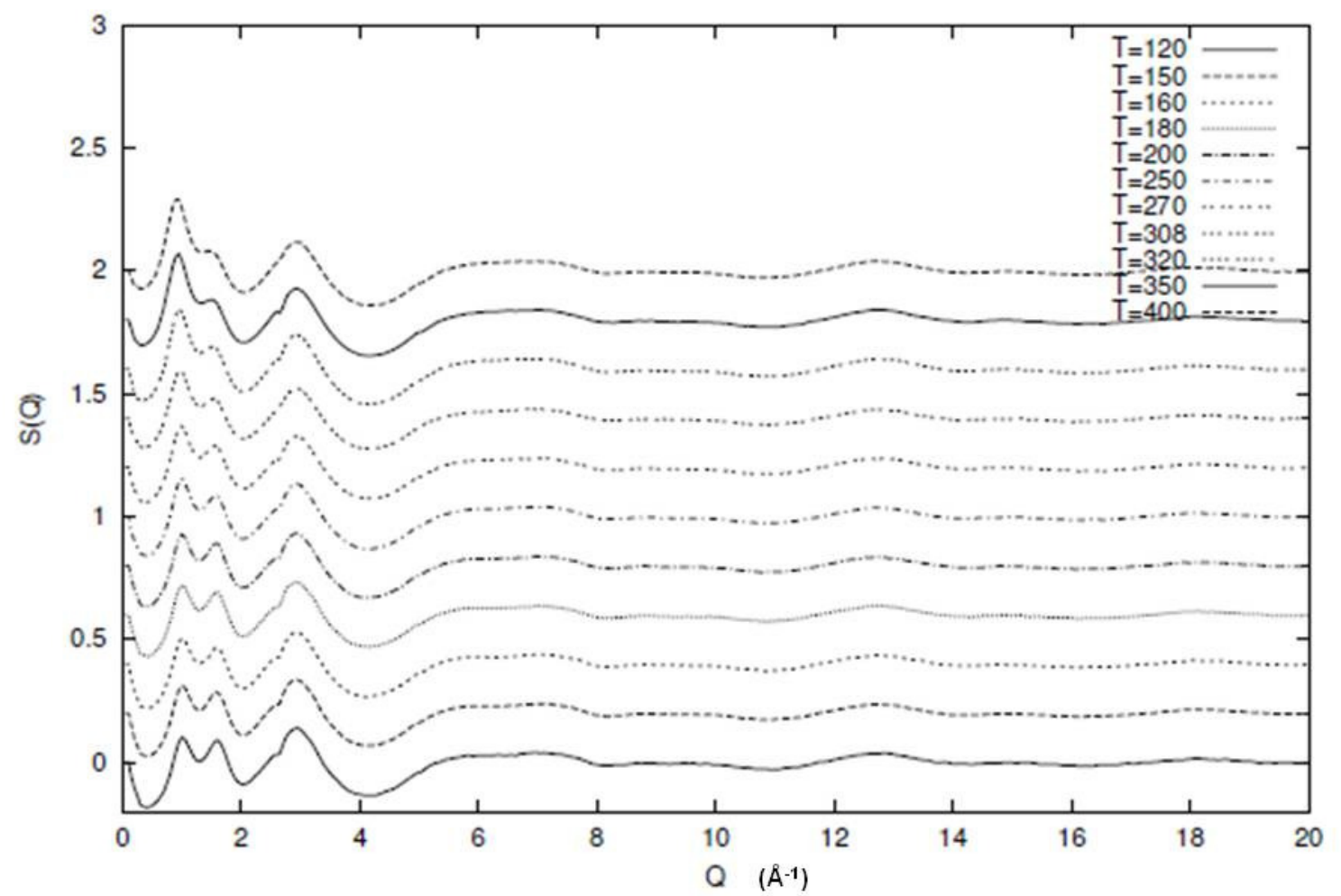

Figure 1. Neutron scattering data for 1,2 polybutadiene as a function of temperature. Differences can be seen in the low $\mathrm{Q}$ region that corresponds to scattering of interchain origin. In the high $\mathrm{Q}$ region the pattern corresponding to the local conformation is virtually unchanged. The curves have been shifted vertically to facilitate the reader.

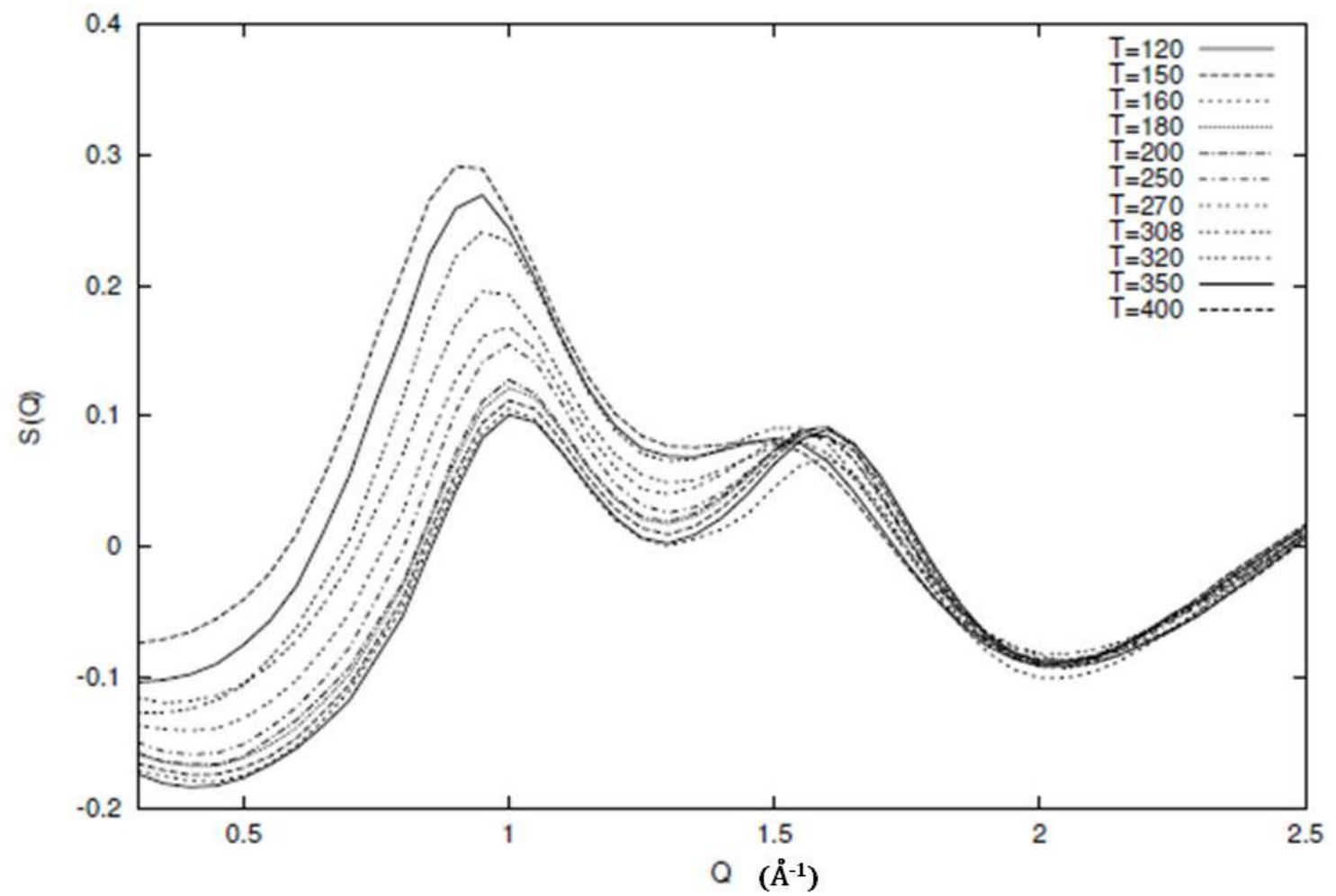

Figure 2 The main scattering peak of 1,2 polybutadiene from neutron scattering data covering the liquid and glassy regime (120-400K). Differences between the structure factors in the low Q region are significant indicating 
differences in segmental mobility and packing in the bulk. The intrachain region at higher $\mathrm{Q}$ values is virtually unchanged.

Similar behaviour has been reported for a number of amorphous polymers[6-13, 34, 35, 38, 39] and is associated with two distinct areas of the diffraction pattern dominated by the intrachain and interchain scattering. The area of the first peak (Figure 2) is dominated by correlations between segments of different or the same chains, highlighting possible changes in the spatial arrangements and packing of the polymer in the bulk. The rest of the diffraction pattern remains almost unchanged with temperature and is dominated by interactions between atoms that are covalently bonded. Consequently the lower sensitivity to temperature that this part of the diffraction exhibits does not come as a surprise. The double intersegmental peak can be a manifestation of the packing of chain segments with the vinyl side groups, something seen before for materials of similar architecture $[6,10,40]$. In fact the two peaks and the strong sensitivity of the first peak to varying temperature is very similar to the behaviour of atactic polystyrene[50]. For polystyrene these features were attributed to the local clustering of the phenyl side groups and a similar interpretation seems reasonable here.

In Figures 3 and 4 the peak positions of the first intense peak of the structure factor as a function of temperature can be seen.

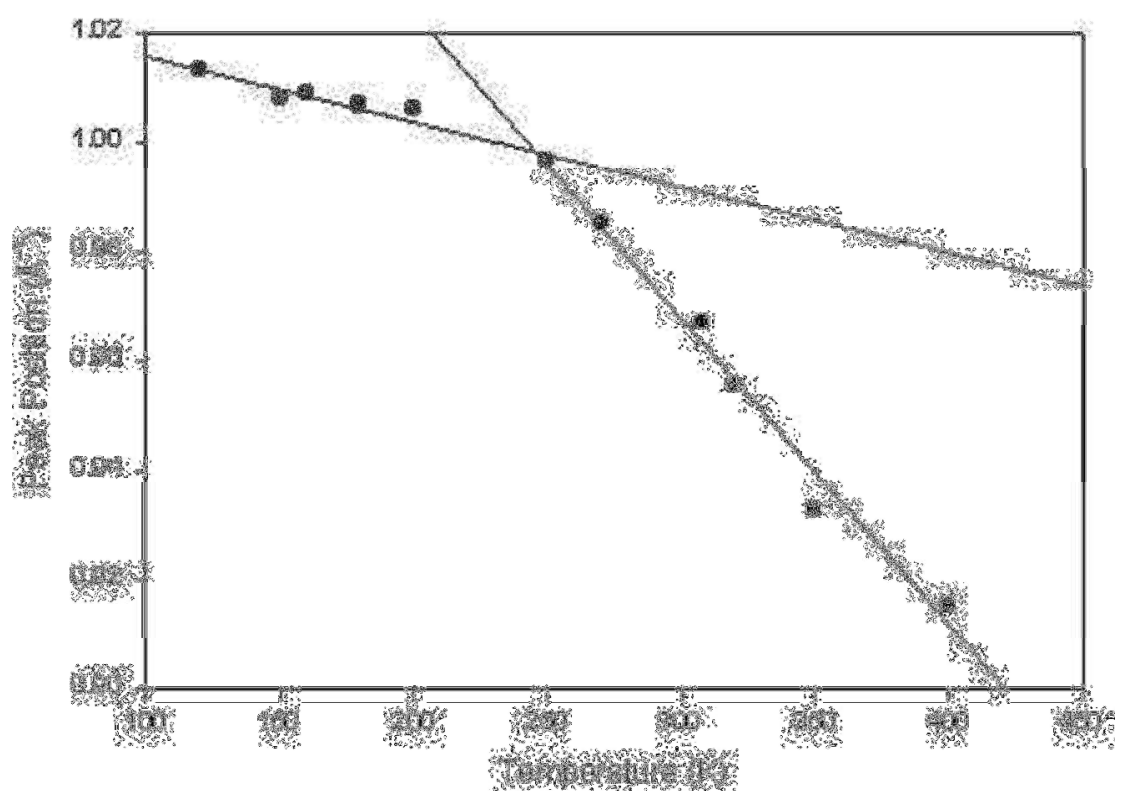

Figure 3 Peak position (points) of the first intersegmental peak of the diffraction pattern of 1,2 polybutadiene as a function of temperature. Liquid and glassy regimes can be identified by the cross-over of the solid lines fitted to the data. 


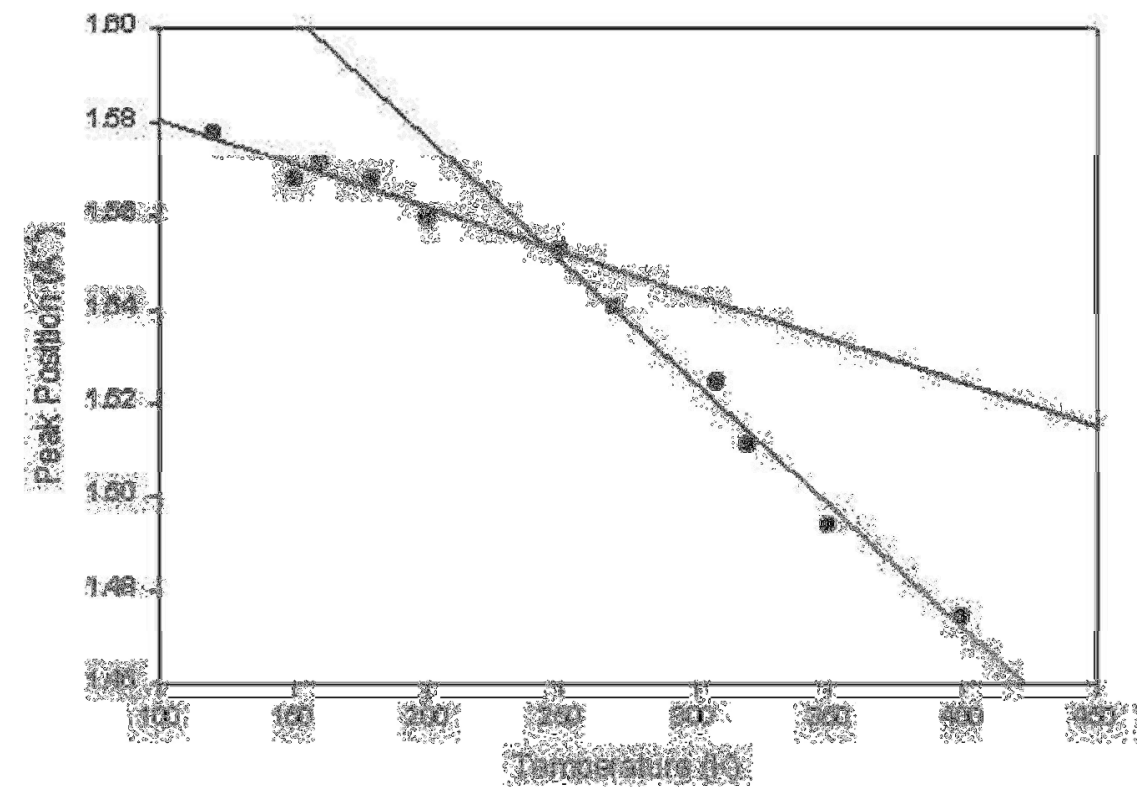

Figure 4 Peak position (points) of the second intersegmental peak of the diffraction pattern of 1,2 polybutadiene as a function of temperature. Liquid and glassy regimes can be identified by the cross-over of the solid lines fitted to the data

From these figures we can see the cross-over between the liquid and glassy regime with the intersection of the fitted lines to the data indicating the vicinity of the glass transition temperature. The value obtained by observation of the diffraction pattern is approximately $250 \mathrm{~K}$, in relative agreement with previously reported values[41-43] and the DSC measurements. In Figure 5 the width of the second interchain peak can be seen. The width of the first peak proved difficult to determine in a reliable manner due to the merging of the two peaks at high temperatures. The position of these intersegmental peaks can be associated with the density of the material hence it is expected to be much denser in the glassy state than in the liquid due to the energetic level of the atoms. As expected in the glassy region the structure is almost frozen with very limited movement indicating a more or less constant density value. Above the glass transition the polymer crosses to the liquid phase with molecular movement performed unrestricted, resulting in a decrease of the density value. The slope of both curves can be associated directly with the thermal expansion coefficient and from the experimental data the values obtained were 5.6 and $5.2 \times 10^{-4} \mathrm{~K}^{-1}$ for the first and second peak respectively.

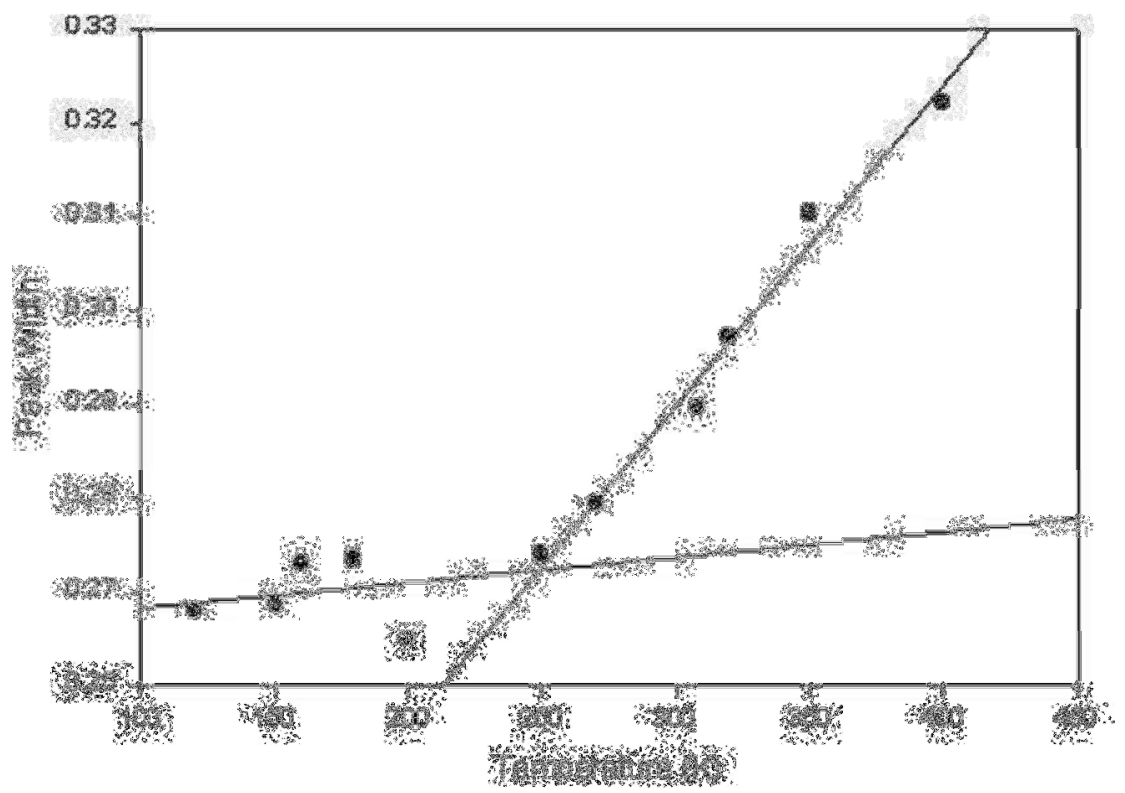

Figure 5 Peak width (points) of the second interchain peak of the diffraction pattern of 1,2 polybutadiene. The solid lines are inserted to help the reader distinguishing between the liquid and glassy regimes.

From the width of the intersegmental peak we calculated the correlation length. The change in position and width of the first two scattering peaks can be associated with the interchain contribution to the polymer. The thermal change in the liquid phase is seen to be much larger than in the glass and by assuming that the width $\sigma$ of the main scattering peak arises from the distribution of near-neighbour distances, the correlation length $\zeta$ can be defined as $\zeta=4 \pi / \sigma$. In 
our case the calculation of the correlation length from the experimental data (Figure 6) is seen to decrease with increasing temperature, and below the glass transition temperature it adopts an almost constant value of approximately $25 \AA$. Our calculation is based on the method developed by Frick, Richter and Ritter[38] and applied in the case of 1,4 polybutadiene with promising results[13].

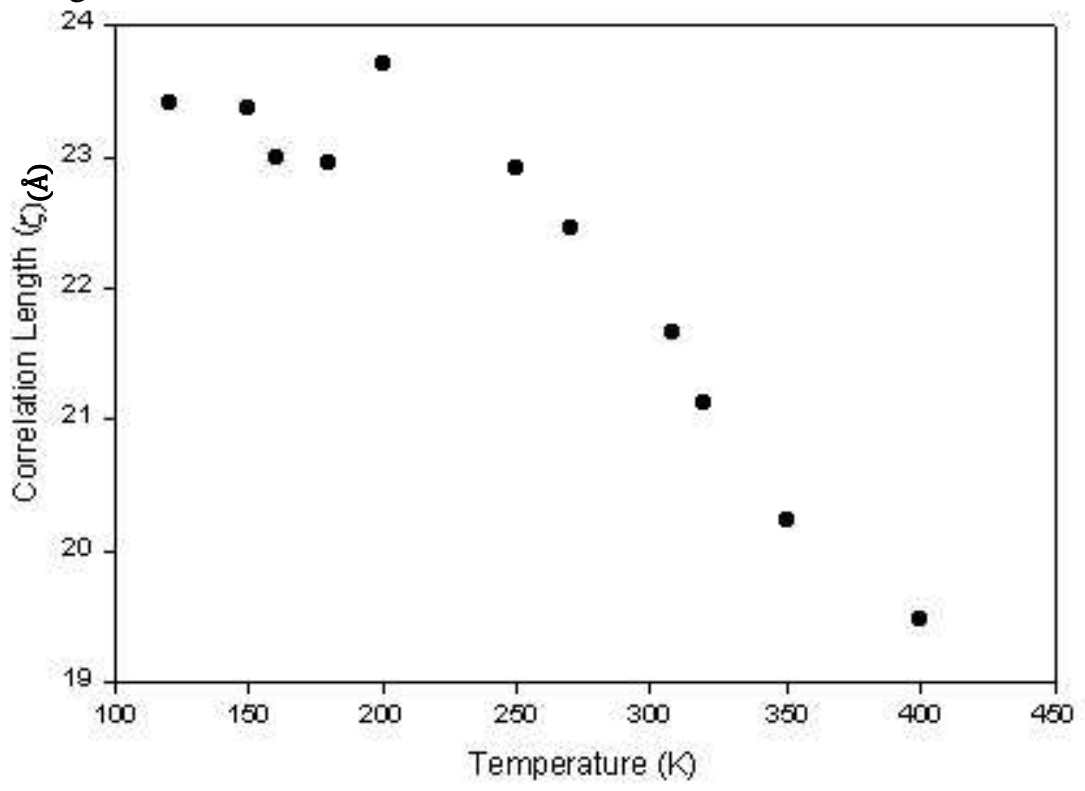

Figure 6 The correlation length $\zeta$ calculated from the width of the main scattering peak of 1,2 polybutadiene as a function of temperature. The different regimes (liquid and glassy) are visible with the value of $\zeta$ increasing with decreasing temperature and adopting an almost stable behaviour below the glass transition temperature.

\section{Experimentally Driven Modelling}

The initial polybutadiene chain was built as a single chain with the linear 1,4 fraction distributed randomly along the backbone according to the percentage obtained from the characteristics of the polymer given in the experimental section. The nature of the search methodology through the n-dimensional parameter space means that the initial set of parameters for the model has no impact on the final structure, something confirmed by earlier studies[13]. Nevertheless aiming to decrease computation time, we used RIS-based values[21, 22] taken from published theoretical models for our initial configuration, and the chain was built with five isomeric states. A threefold potential with minima at trans and $s \pm$ with torsion angles at $0^{\circ}$ and $\pm 120^{\circ}$ for the two backbone torsion angles, $\alpha_{1}$ and $\alpha_{2}$, and a vinyl potential with minima at $g \pm$, cis and trans with torsion angles at $\pm 60^{\circ}, 180^{\circ}$ and $0^{\circ}$ (see Illustration).

The 11 patterns at different temperatures are treated as independent measurements and the structural refinement procedure is performed individually. The final value of the statistical test for each of the experimentally observed structure factors was $0.34 \pm 0.02$, giving confidence on the technique's output, especially if one considers the large number of data sets utilised. Fluctuations of the statistical test output are associated with the level of tolerance of the test and the data quality.

A number of models were built for any given parameter set to the relevant value keeping all other model parameters constant. The resulting diffraction was compared with the observed structure factor, and the calculation continued until convergence was achieved (within the desired level of accuracy of the relevant parameter). The best fit value of the investigated parameter was extracted as the one providing the minimum $\chi^{2}$ test result and thus the best fit between model and experiment. The procedure was repeated for all parameters, after the previous parameter was fixed to the value extracted by the $\chi^{2}$ test, and the overall search was repeated as many times as it was necessary to achieve an overall convergence in the $\chi^{2}$ values. In order to have good statistics and allow for the proper representation of all relevant configurations we used an average of 10 models (15,000 skeletal bonds) for the bond lengths, 50 models for the valence angles and 100 models for the identification of the rotational conformational characteristics. In Figure 7 the $\chi^{2}$ test result for a given parameter (the $\mathrm{D}-\mathrm{C}=\mathrm{C}$ valence angle in this case) is presented indicating the vicinity of the minima where the parameter minimises the difference between the observed and calculated scattering functions. 


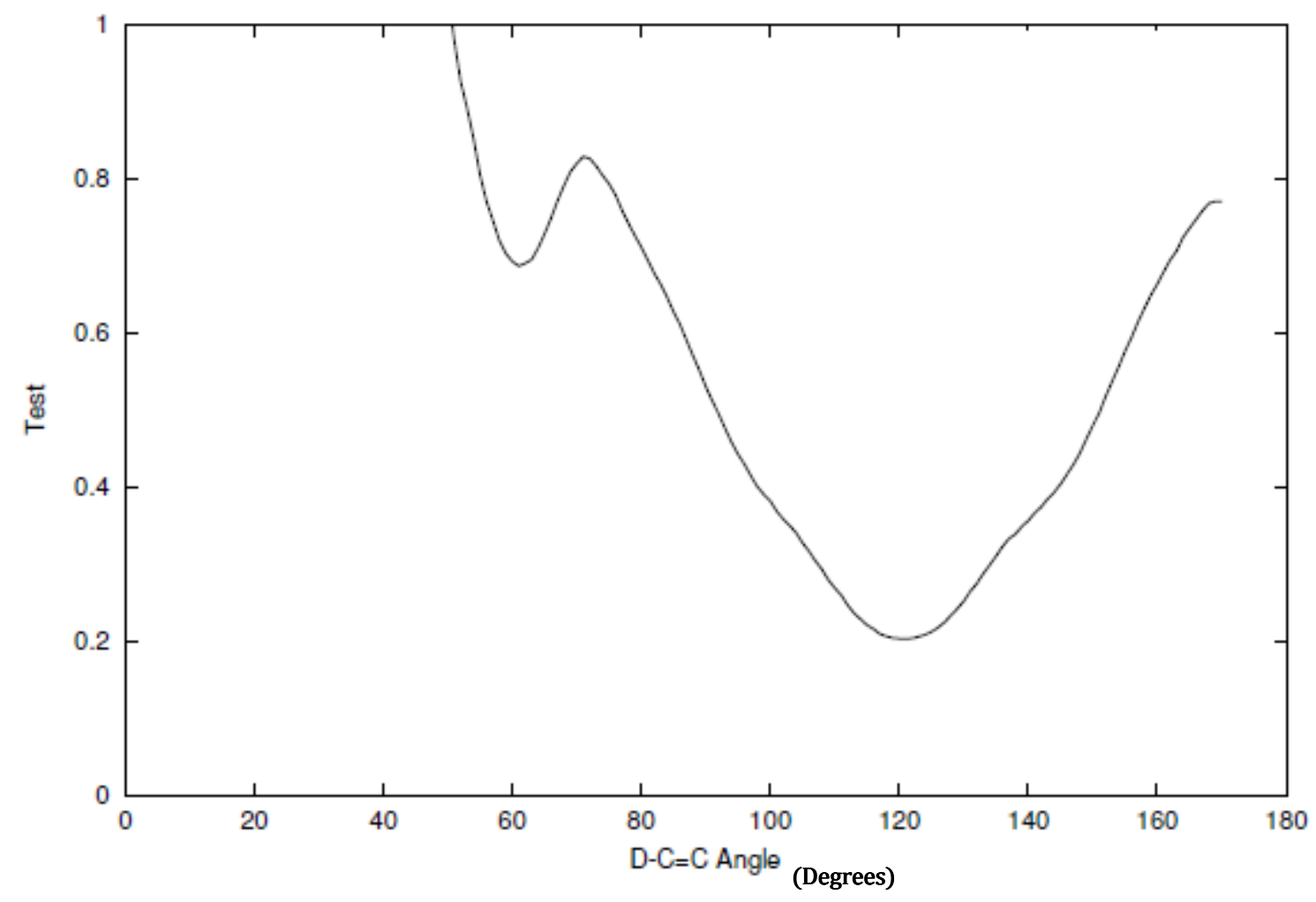

Figure $7 \chi^{2}$ test result for the $\angle \boldsymbol{D}-\boldsymbol{C}=\boldsymbol{C}$ valence angle indicating the vicinity of the minima.

\section{Bond Lengths and Angles}

Table 1 summarises the results obtained from this study along with previously reported results on crystalline compounds[44-46] and theoretical predictions[21, 23]. For the entire temperature range (120-400K) the bond length positions of the distributions defining the $\mathrm{C}-\mathrm{D}, \mathrm{C}-\mathrm{C} . \mathrm{C}=\mathrm{C}, \mathrm{C}-\mathrm{C}=$ and $=\mathrm{C}-\mathrm{C}$ bonds were found to be fixed at a single value, and were identified with high level of accuracy of the order of $\pm 0.01 \AA$. The widths of the distributions were found to depend heavily on the data quality (especially in the high scattering vector region) and were almost invariant with temperature, contrary to expectations that there would be a small change due to thermally stimulated vibrations. This effect is also seen previously[13] and originates from the instrumental resolution. It is worth noting the greater precision achieved in a much earlier study of molten polytetrafluoroethylene[9], where the data had been collected using a now defunct instrument of greater resolution and for a polymer in which the intrachain structure was more ordered. Thus as well as the noise which will be present in the real experimental data, the resolution in the data analysis will be influenced by the instrument used and by the extent of the intrachain order.

In Figure 8 the positions of the valence angles distributions for the entire temperature range is presented, and in Table 1 the average value for each valence position is presented along with previously reported results. The positions of the valence angles distribution were found to be in good agreement with computer simulations[23], experimental data[44, 45] and RIS predictions[21]. The level of accuracy of the position obtained is of the order of $0.5-1^{\circ}$, and unlike the bond lengths the positions of the distributions of the valence angles exhibit a slight temperature variation. This existent small level of fluctuation can possibly be attributed to a slight level of anharmonicity of the valence potential. 


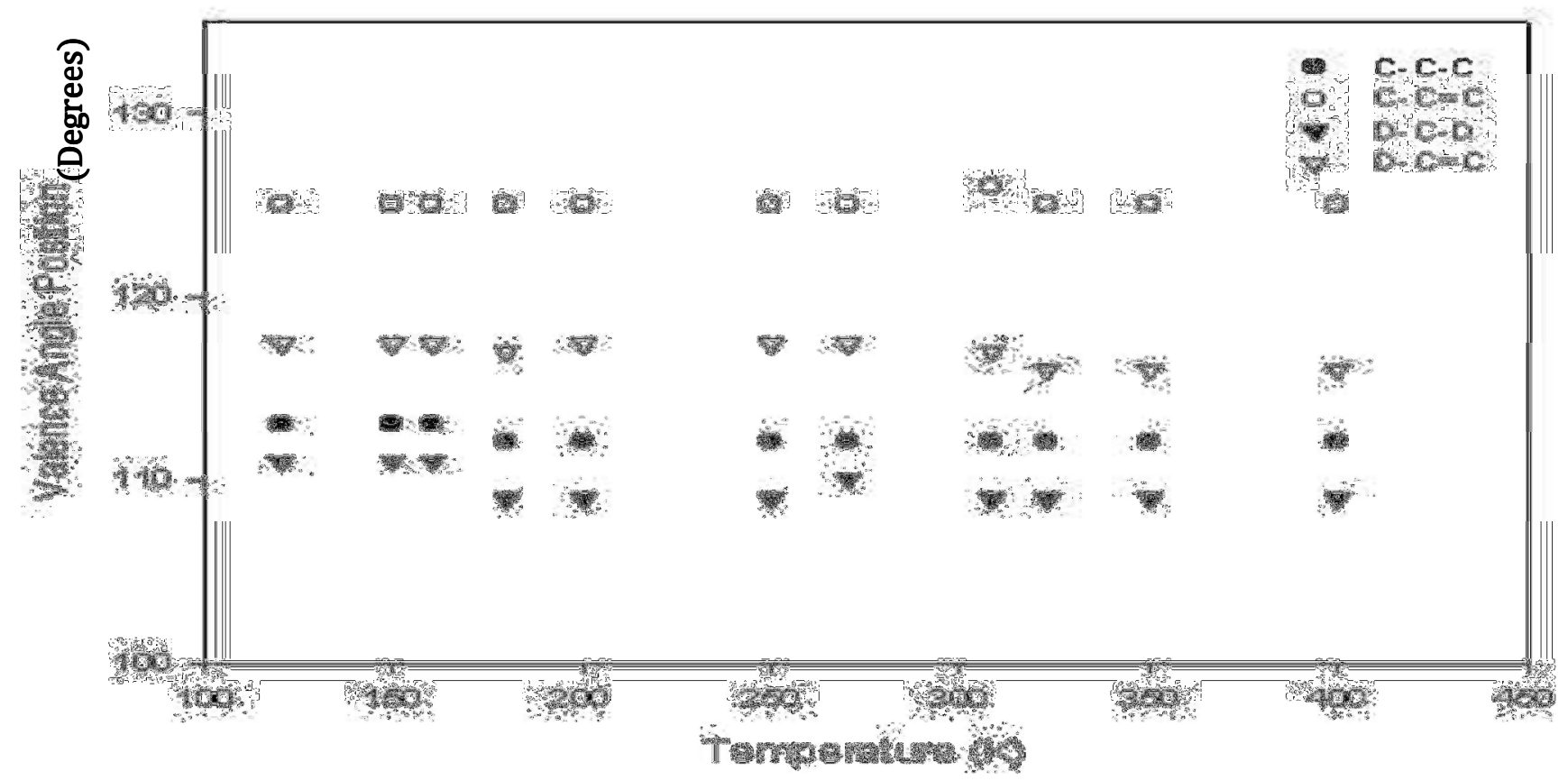

Figure 8 Valence angle distributions' peak positions for the different configurations along the 1,2 polybutadiene chain as a function of temperature. In the entire experimentally accessible temperature range the valence angles position appears to be almost constant with negligible fluctuations of the order of a few degrees.

\begin{tabular}{|c|c|c|c|c|}
\hline Model Parameter & This Work & $\mathbf{M c} \mathbf{c}^{\mathbf{a}}$ & Crystalline $^{b}$ & RIS $^{\mathbf{c}}$ \\
\hline $\mathrm{C}-\mathrm{C}$ & $1.54 \pm 0.01 \AA$ & $1.53 \AA$ & $1.53 \AA$ & \\
\hline $\mathrm{C}-\mathrm{C}=$ & $1.51 \pm 0.01 \AA$ & $1.51 \AA$ & $1.51 \AA$ & \\
\hline C-D & $1.13 \pm 0.01 \AA$ & & $1.1 \AA$ & \\
\hline $\mathrm{C}=\mathrm{C}$ & $1.35 \pm 0.01 \AA$ & $1.34 \AA$ & $1.34 \AA$ & \\
\hline $\mathrm{C}-\mathrm{C}-\mathrm{C}$ & $112.27^{\circ} \pm 1^{\circ}$ & $111.65^{\circ}$ & $112.1^{\circ}$ & $112^{\circ}$ \\
\hline $\mathrm{C}-\mathrm{C}=\mathrm{C}$ & $125.09^{\circ} \pm 1^{\circ}$ & $125.89^{\circ}$ & $125^{\circ}$ & $120^{\circ}$ \\
\hline $\mathrm{D}-\mathrm{C}=\mathrm{C}$ & $117^{\circ} \pm 1^{\circ}$ & & $119^{\circ}$ & $117.5^{\circ}$ \\
\hline D-C-C & $109.63^{\circ} \pm 1^{\circ}$ & & & \\
\hline$\alpha_{1}$ bond & trans, $\pm 120^{\circ}$ & $\operatorname{trans}, \pm 120^{\circ}$ & $\operatorname{trans}, \pm 120^{\circ}$ & trans, $\pm 120^{\circ}$ \\
\hline$\alpha_{2}$ bond & trans, $\pm 120^{\circ}$ & trans, $\pm 120^{\circ}$ & trans, $\pm 120^{\circ}$ & trans, $\pm 120^{\circ}$ \\
\hline vinyl bond & $\pm 60^{\circ}$ & cis, $\pm 60^{\circ}$ & cis, $\pm 60^{\circ}$ & $c i s, \pm 60^{\circ}$ \\
\hline
\end{tabular}

Table 1 Comparison between the structural parameters obtained for 1,2 polybutadiene with previously reported experimental data and theoretical predictions. a (Ref. 23), b (Ref. 44-46), c (Ref. 21)

\section{Torsion Angles}

Positions of the distributions of the two backbone torsion angles $\alpha_{1}$ and $\alpha_{2}$ were found occupy trans and $s \pm$ states at $0^{\circ}$ and $\pm 120^{\circ}$, experimentally confirming previously reported predictions[21-24], and energy calculations of crystalline compounds[44-46]. The position of the distribution of each torsion population was located in a well defined minimum with a level of uncertainty of the order of $2-3^{\circ}$ for all temperatures. The width of the distribution from the refinement procedure was found in a clear, well localised minimum in the region of $4-10^{\circ}$ depending on the temperature, with a level of uncertainly of the order of $2-3^{\circ}$. The picture emerging from the refinement technique is that of fluctuations around the mean of the distribution for the torsion angles are frozen in the glassy state (120-250K). In the liquid state (250-400K) a rapid expansion of the width of the distribution of the torsion populations can be associated with the increased level of mobility and thermal fluctuations in the liquid state.

The $\alpha_{1}$ torsion angle was found to occupy the trans state almost exclusively for the whole temperature range at a fraction of $90-95 \%$ with a level of uncertainty of the order of $\pm 10-15 \%$. This experimental behaviour differs from observed Monte Carlo[23] and Molecular Dynamics[22] simulations of atactic and isotactic 1,2 polybutadiene that predict a threefold potential for the backbone with approximately 60-40\% trans-skew ratio in the melt. The $\alpha_{2}$ bond was found to have a trans fraction that changes as temperature rises from approximately $20 \%$ to $40 \%$ with a level of uncertainty of the order of $\pm 10 \%$. This behaviour is in very good agreement with previously reported quantum mechanical predictions[24] as can be seen from Figure 9. 
The vinyl angle was found in this work at $\pm 60^{\circ}$ in good agreement with previously reported predictions[21-24]. One set of Monte Carlo simulations[23] reported a small population occupying the trans state, but the introduction of such possibility in this work did not lead to a reduction in the statistical test and was not carried forward in the model.

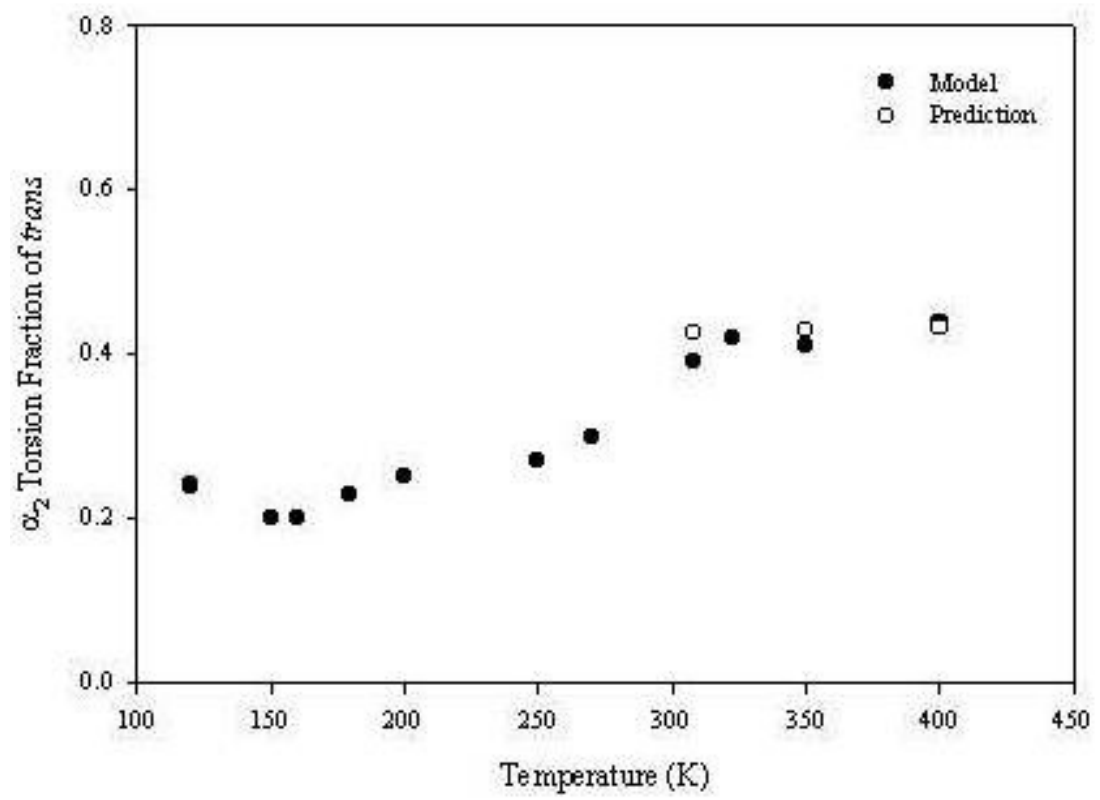

Figure 9 Fraction of trans of the $\alpha_{2}$ bond for 1,2 polybutadiene extracted using the refinement technique (solid circles) as a function of temperature. The comparison with previously reported quantum mechanical predictions[24] (open circles) in the melt is very good.

\section{Characteristic Ratio}

This study is focused on the local structure and some of the local fluctuations will cancel over a larger distances. Larger scale properties such as the characteristic ratio $C_{\infty}$ can be estimated from the polymer chain models used in this study. Clearly any realistic model must match the experimental observations on a range of length scales. A series of optimised models (approximately 1000) for each temperature were generated, creating a set of stochastically built chains of similar local structure. The mean squared end to end length for each chain was calculated, and the characteristic ratio of the large chain was extracted using the formula $C_{n}=\left\langle\mathbf{r}^{2}\right\rangle_{o} / \sum_{i=1}^{X-1} l_{i}^{2}$, where X-1 is the number of links in the backbone and $l^{2}$ the average squared skeletal bond length. For 1,2 polybutadiene $\sum_{i} l_{i}^{2}=1.54 \AA$. The characteristic ratio (Figure 10) has an average value of 8.9 with a level of uncertainty of the order of \pm 0.5 over the temperature range considered. $\Theta$ solvent measurements of essentially 1,2 polybutadiene with just $2 \%$ of 1,4 polybutadiene[47] as part of a broader study of polydiens and polyolefins give a value for $C_{\infty}$ of 7.7 in relative agreement with the values obtained in this work using the optimised local structure. RIS predictions[48] indicate a value of 9.5 for pure 1,2 polybutadiene. 


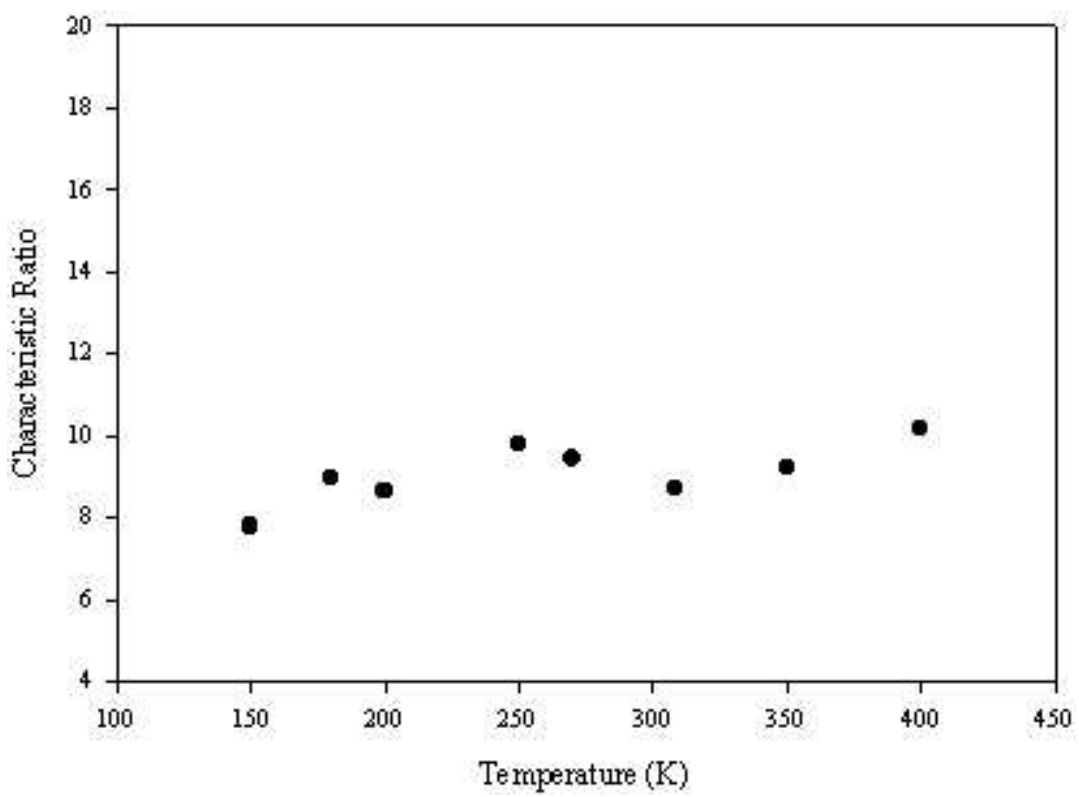

Figure 10 The Characteristic ratio of 1,2 polybutadiene obtained from the optimised models of the refinement technique as a function of temperature.

Solvent measurements[47] indicate a reduction in the value of $C_{\infty}$ with a temperature coefficient of $-1.7 \times 10^{-3} \mathrm{~K}^{-1}$, which might be attributable to the enhancement of the skew population of the backbone caused by non-bonded interactions of the side group. Our study yields a small rate of change in the characteristic ratio which is of opposite sign. At the local level we relate this to the observation that the $\alpha_{1}$ bond adopts an almost complete trans conformation over the entire temperature range. These variations underline the different conformational behaviour of the 1,2 and 1,4 units. Of course in this work the system is a polymer melt and the literature values come from the solution state albeit in the theta state. The differences may simply reflect that the melt chain conformation is slightly more persistence than that observed in solution as consequence of the clustering of the side groups as revealed by the strong temperature effects of the scattering shown in Figure 2.

Monte Carlo[23] and quantum chemistry calculations[24] predict conformational characteristics which would lead to a lower value of $C_{\infty}$, as seen with the $\Theta$ solvent measurements. The results presented here are in relative agreement with the predictive and experimental results. The difference may be as suggested above or due to a limitation in the neutron scattering analysis in that we observed some differences in the characteristics of the $\alpha_{1}$ bond with regard to gauche states with theoretical calculations.

In the procedure used here the 1,4 polybutadiene units were treated independently in a way similar to the 1,2 counterpart, without taking into consideration possible conditionality of the rotational states confined between two double bonds. 1,4 polybutadiene has been seen to affect the chain dimensions in different ways depending on the state of the double bond (cis or trans) and the specific couplings of the $\alpha$ and $\beta$ bonds adjacent to it[49]. Since the fraction of 1,4 component in our system is significant the lack of conditionality on the torsion states is expected to have an effect on the chain dimensions, something seen already in a previous study[13].

\section{Diffraction Pattern}

In this study the whole refinement procedure is dependent on comparisons between the experimentally observed and the computer-generated structure factors. Therefore one expects that a certain level of association of the different model parameters with different areas or special features should be possible. Obviously all parameters affect the entire intrachain region, but sub-sections of the scattering curve that are more sensitive to the optimisation of parameters of different length scales do exist. In this work we have observed that the scattering originating from atoms joint together via covalent bonds (especially the C-D bonds) have the most profound effect on the overall shape of the scattering curve, something seen before in the case of linear polybutadiene[13] and other polymers[34,35]. This effect although it is visible in the entire scattering curve, manifest itself more in the high $Q$ region $\left(\mathrm{Q}>20-25 \AA^{-1}\right)$. The low $Q$ region is mostly dominated by parameters that affect the valence and torsion angles, with the later having almost no effect on the shape of the high $Q$ area. In Figure 11 a schematic representation of the different parameters on the structure factor can be seen, together with the best fit model and the experimentally observed structure factor. 


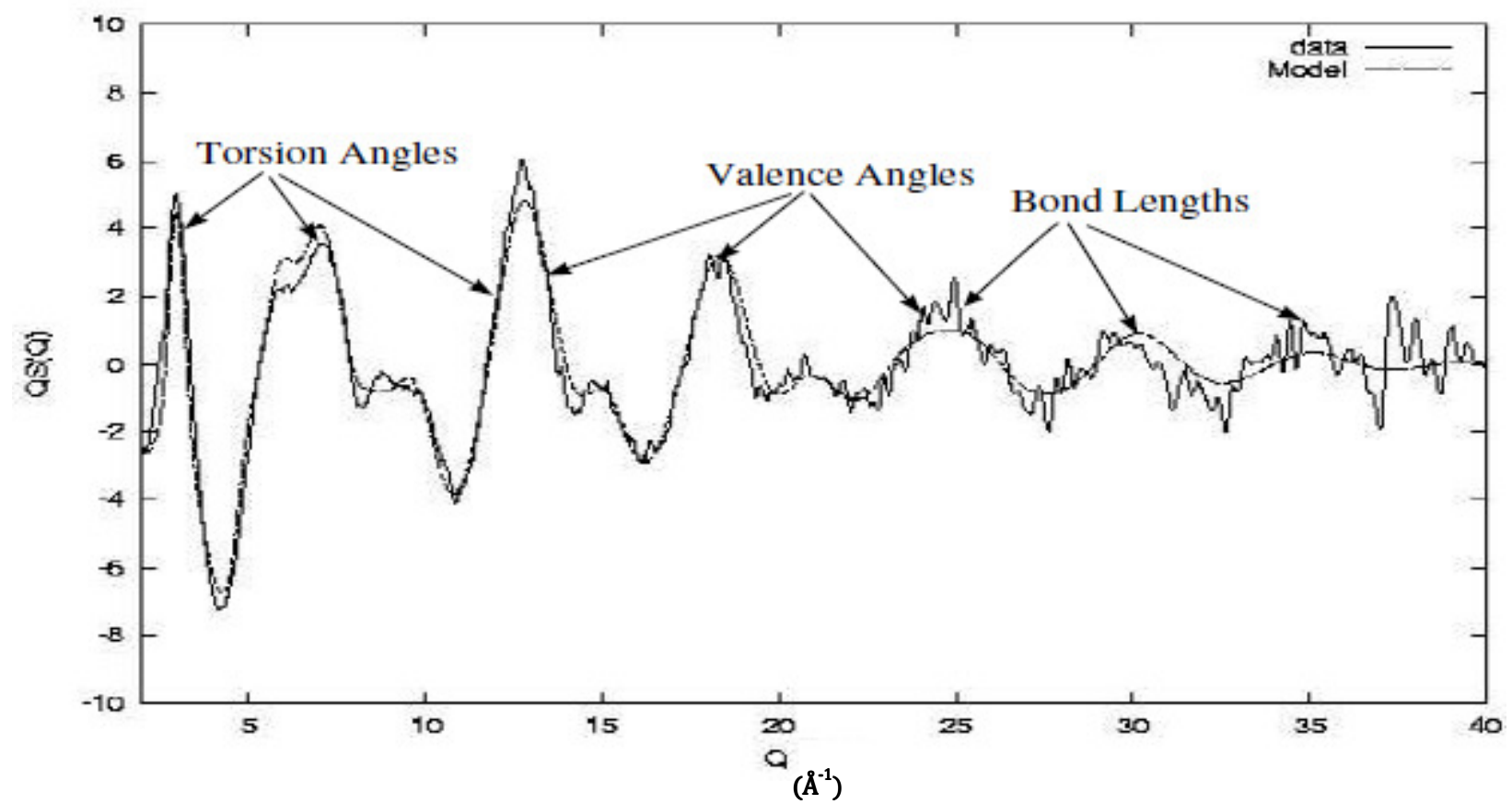

Figure 11 Schematic Comparison of the experimentally observed structure factor (data - solid line) of 1,2 polybutadiene and the best fit from the structural refinement procedure (model - dotted line). The different regions of the scattering are also presented along with the different types of structural parameters that affect them the most.

\begin{abstract}
Summary
In this study we have used high quality neutron scattering data over an extended $Q$ range, intimately coupled with stochastically built atomistic models for detailed structural analysis of amorphous polymers. We have shown that fully deuterated polymers combined with the high sensitivity of neutrons can offer unique possibilities of extracting intrachain correlations from a relatively simple statistical model by direct comparison with the experimentally observed structure factor. Thus information on the structural characteristics of the repeat unit and on the degree of stiffness of the polymer under investigation is possible.
\end{abstract}

Broad $Q$ neutron scattering patterns covering most of the experimentally accessible temperature range (120-400K) including both liquid and glassy regimes for atactic 1,2 polybutadiene have been presented. Each of the 11 temperatures used can be seen as an independent measurement, used to validate the experimentally-driven modelling procedure while providing information on the chain conformation of the material at the given state (temperature). The interchain region of the scattering pattern exhibits differences in terms of both position and width of the main (double) scattering peak with temperature. These differences are attributed to density changes and the system's dynamics. In the glassy regime these changes are very small, indicating a frozen configuration that allows only minimal molecular motion. In the liquid regime where molecular motion is unrestricted changes are much more significant. The intrachain region of the scattering pattern appears to be almost insensitive to temperature for both liquid and glassy states. From the main scattering peak we have calculated the correlation length that adopts an almost stable value of approximately $25 \AA$ in the glassy region.

Reciprocal space analysis based on the minimisation of differences between the experimentally observed diffraction pattern and the one calculated from an atomistic model has been presented. Different length scales have been probed independently allowing us to observe their effect on the specific regions and shapes of the structure factor. Bond lengths and valence angles are found to be in almost total agreement with previously reported predictions and experimental findings. From our study we have concluded that normal distributions for modelling bond lengths and angles offer high accuracy, indicating an almost totally harmonic potential for the bond lengths and a slight anharmonicity of the valence angle distribution. Local conformation indicates a set of two backbone torsional angles adopting trans and skew states. Their behaviour is totally different, probably due to non-bonded interactions caused by the rotation of the vinyl groups. These interactions would probably enforce higher order probabilities in the states available to the backbone since strong coupling between neighbouring states is expected. Our approach assumes no such coupling, so a certain level of overcompensation between the different torsion angles cannot be disregarded. The $\alpha_{1}$ bond obtains an almost insensitive to temperature high trans conformation, something not seen in previously reported simulations and theoretical predictions. The $\alpha_{2}$ bond adopts an almost equally distributed threefold configuration, with the trans fraction increasing with temperature in good agreement with previously reported theoretical predictions. The vinyl angle adopts $\pm 60^{\circ}$ conformation equally weighted in good agreement with 
previously reported simulations. A small population of trans predicted by Monte Carlo simulations in the past was not found in this study. Extrapolation of large scale properties like the characteristic ratio is in relative agreement with previously reported RIS predictions and experimental measurements. Based on these results we can see a picture of a relatively stiff 1,2 polybutadiene chain, something attributed mostly to the high trans sequences of the backbone.

\section{Acknowledgments}

This work was supported by the University of Reading and the Science and Engineering Research Council. We would like to acknowledge CCLRC for providing access to ISIS neutron facility, thank Alan Soper and Daniel Bowron for all their help and support during the neutron scattering experiments and Fred Davis, Robert Olley and Clark Balague for all their help with the sample preparation and characterisation.

\section{References}

1. Volkenstein M. V. Configurational Statistics of Polymer Chains, New York, Wiley-Interscience, 1963.

2. Flory P. J. Statistical Mechanics of Chain Molecules, New York, Wiley-Interscience, 1969.

3. Lovell R., Mitchell, G. R. and Windle, A. H., Faraday Discuss. Chem. Soc. 68, 46 (1979)

4. Frick, B. and Richter, D. Science 267, 1939 (1995)

5. Richter, D. Physica B 276-278, 22 (2000)

6. Mitchell, G. R. in Order in the Amorphous State of Polymers Eds. Keinath S. E. Miller R. L. Rieke J. K. Plenum, New York, 1987.

7. Mitchell, G. R. in Comprehensive Polymer Science Eds. Allen G. Bevington J. C. Booth C. Price C. Pergamon, Oxford, 1989.

8. Mitchell G. R. and Rosi-Schwartz B. Physica B 180, 558 (1992)

9. Rosi-Schwartz B. and Mitchell G. R., Polymer, 35, 3139 (1994)

10. Mitchell G. R., Rosi-Schwartz B. and Ward D. J., Phil. Trans. R. Soc. Lond. A, 348, 97 (1994)

11. Rosi-Schwartz B. and Mitchell G. R., Nucl. Instr. And Meth. In Phys. A, 354, 17 (1995)

12. Rosi-Schwartz B. and Mitchell G. R., Physica Scripta, T57, 161 (1995)

13. Gkourmpis T. and Mitchell G. R., Macromolecules, 44, 3140 (2011)

14. McGreevy R. L. and Pusztai L., Mol. Sim., 1, 359 (1988)

15. Chanda, M. and Roy, S. K. Plastics Technology Handbook, CRC Press, New York 2007.

16. Solomon, G. Discuss. Faraday Soc. 2, 361 (1947)

17. Natta, G. and Corradini, P. Angew. Chem. 68, 615 (1956)

18. Mark, J. E. J. Am. Chem. Soc. 88, 4354 (1966)

19. Deegan, R. D. and Nagel, S. R. Phys Rev B, 52, 5653 (1995)

20. Arbe, A. Richter, D. Colmenero, J. and Farago, B. Rhys Rev E, 54, 3853 (1996)

21. Ma, H. and Zhang, L. Polym. J. 26, 121 (1994)

22. Kim, E. Misra, S. and Mattice, W. L. Macromolecules 26, 3424 (1993)

23. Getoso, P. Nicol, E. Doxastakis, M. and Theodorou, D. N. Macromolecules 36, 6925 (2003)

24. Smith, G. D. Paul, W. Monkenbush, M. Willner, L. Richter, D. Qiu, X. H. and Ediger, M. D. Macromolecules 32, 8857 (1999)

25. Birshtein, T. M. and Pitisyn, O. B. Conformations of Macromolecules, Interscience, New York 1966

26. Mitchell G. R. MESA, A Molecular Editor for Structural Analysis, Reading UK 1996

27. Flory P. J. J. Chem. Phys. 17, 303 (1949)

28. Warren B. E. X-Ray Diffraction, Addison-Wesley, Reading, MA 1969

29. Mitchell, G. R. Lovell, R. and Windle A. H. Polymer 23, 1273 (1982)

30. Squires, G. L. Introduction to the Theory of Thermal Neutron Scattering, Dover, London, 1996

31. Windsor, C. G. Pulsed Neutron Scattering, Taylor \& Francis, London, 1981

32. Turner, J. Z. Soper, A. K. Howells, W. S. Hannon, A. C. and Ansell, S. SANDALS Survival Guide version 2.5, Rutherford-Appleton Laboratory, ISIS Facility, Chilton, 1995.

33. Bevington P. R. Data Reduction and Error Analysis for the Physical Sciences, McGraw-Hill, New York, 1969

34. Mitchell G. R. in Essentials of Neutron Techniques in Soft Matter, Eds. Imae T., Kanaya T., Furusaka T., Willey 2010

35. Gkourmpis T., Lopez D. and Mitchell G. R., 2012 Mater. Res. Soc. Symp. Proc., 1524, (2013)

36. Benmore, C. J. and Soper, A. K. RAL Technical Report RAL-TR-1998-006, Rutherford-Appleton Laboratory, ISIS Facility, Chilton, 1998

37. McLain S. E., Bowron D. T., Hannon A. C. and Soper A. K. Gudrun A Computer Program Developed for Analysis of Neutron Diffraction Data, ISIS Facility, Rutherford-Appleton Laboratory, UK 2012

38. Frick B. Richter B. and Ritter C. Europhys. Lett. 9, 557 (1989)

39. Mitchell G. R. in Concise Encyclopaedia of the Structure of Materials Ed. Martin J. W.; Elsevier, London 2007

40. Mitchell G. R. and Chiou Y-S. Advances in X-Ray Analysis 41, 640 (1997) 
41. Carpenter R. L. Kramer O. and Ferry J. D. Macromolecules, 10, 117 (1977)

42. He T. Li B. and Ren S. J. Appl. Polym. Sci., 31, 873 (1986)

43. Ni S. Yu F. Shen L. and Qian B. Chin. J. Polym. Sci., 5, 120 (1987)

44. De Rosa C. Zhi G. Napolitano R. and Pirozzi B. Macromolecules, 18, 2328 (1985)

45. Corradini P. Napolitano R. Petraccone V. Pirozzi B. and Tuzi A. Macromolecules, 15, 1207 (1982)

46. Corradini P. De Rosa C. Zhi G. Napolitano R. and Pirozzi B. Eur. Polym. J., 21, 635 (1985)

47. Hattam P., Gauntlett S., Mays J. W., Hadjichristidis N., Young R. N. and Fetters L. J. Macromolecules 24, $6199(1991)$

48. Jang J. H. Halioglu T. von Meerwall E. D. and Mattice W. L. Macromolecules 33, 4271 (2000)

49. Smith G. D. and Paul W. J. Phys. Chem. A, 102, 1200 (1998)

50. Mitchell G. R. and Windle A. H. Polymer, 25, 906 (1984) 